\title{
Causal modeling applied to psychonomic systems simulation
}

\author{
J. JACK MCARDLE \\ University of Denver, Denver, Colorado 80210
}

\begin{abstract}
Psychometric principles in structural equation model building (e.g., LISREL) are applied to the psychonomic problems in simulating causal systems. A new approach is described (i.e., RAM) that permits the complete and concise description of causal logic by nomographicdiagrammatic representation. Several simple examples based on traditional data-analytic models are causally explicated. Relevant numerical algorithms for multivariable simulation in an on-line environment are described. As an illustration of complete structure-process modeling, a substantive example of "human ability systems" is presented. Finally, a broader view of both practical and theoretical applications is offered.
\end{abstract}

This paper represents an attempt to dress up some new psychometric methods in old psychonomic clothes. The broad general question to be dealt with here is this: "If the causal laws of mental activity were operating in a specific way, then what should observed data look like?" A formal answer to this question is provided by using relatively old psychonomic notions about causal laws as the building blocks for the relatively new psychometric mathematics of structural diagrams. These in turn are applied to the possibilities of on-line simulation of data with known causal composition.

This approach promises to be useful in a number of ways. The major theoretical benefit is the illustration of a simple theoretical-statistical tool that comprehensively integrates many forms of psychological theory, design, and analysis. In this psychonomic forum, the goal is to provide a simple introduction to the flexibility of these psychometric ideas. The major practical utility discussed here is a direct and simple way to simulate multivariable systems. Of course, this on-line computerbased approach already has a variety of well-known pedagogic and research uses in psychonomy.

Of central concern are the most recent developments in the techniques of linear structural equation model building (e.g., LISREL; Jöreskog \& Sörbom, 1978; McDonald, 1979). These methods combine and refine many statistical aspects of: (1) general linear model analysis of variance, (2) factor analysis, and (3) path analysis. Due to this historical context, they are usually discussed as empirical tools for theory evaluation by statistical hypothesis testing. Consequently, their use in inferential data analysis is sometimes termed "causal

I wish to thank John L. Horn and Richard S. Lehman for their stimulation and assistance in the development of the ideas presented here. I am also grateful for the helpful comments of Herman O. Wold and Steven E. Poltrock. Funding for this research was provided by John L. Horn under NSF-RIAS Grant SER-77-06935 and NIA Grant R01-AG0058302. modeling" (e.g., Bentler, 1978; cf. Horn \& McArdle, 1979). However, the only question that may be answered by any inferential analysis is this: "Given these data, what is the probability that causal laws are operating in this specific way?" This inferential question is not a direct concern of this presentation.

The recent integration of some of these tools under reticular analytic modeling (RAM) theory (McArdle, 1979, Note 1) alternatively emphasizes the great flexibility of these methods for the formal expression of causal theory. The generic features of RAM illustrate both the flexibility and the limitations of structural modeling for causal inference more easily than these other, seemingly more complex forms. All of these ideas may be presented by reference to the communicable simplicity of RAM nomographs, which are specialized versions of pathdiagram/linear-flowgraph logic (Rapoport, 1972; Wright, 1934). A discussion of the basic elements of RAM mathematics applied to causal systems is presented by several detailed examples.

The multivariable simulation of such mathematical models is presented only from the perspective of generating sample means, variances, and correlations from hypothesized population models (i.e., plasmodes). The use of general systems logic here emphasizes the mathematical creation of static state structures combined with corresponding dynamic state processes (Rozeboom, Note 2). Relevant numerical algorithms for this approach are described and a substantive example from recent research on "human ability systems" (Horn \& McArdle, 1979) is presented. Some important differences between this complete functional/stochasticevent approach and the more prevalent deterministic/ discrete-event simulation of behavior (cf. Klahr, 1976; Lehman, 1977) are also discussed.

One note of caution: do not be discouraged by new technical jargon that may at first seem obscure. Find solace in the fact that the graphics are equivalent to the algebraics. Recent experience suggests that this pictorial 
view definitely provides a more convenient introductory tool. The matrix algebra is only included for its practical utility in the simulation enterprise. Therefore, those unfamiliar with the mathematical mechanics are encouraged to read around the equations, as it were, and to try to extract as much figural representation as possible. On a second pass, the equations should prove much more meaningful and should provide another useful form of communication.

\section{A MATHEMATICAL REPRESENTATION OF CAUSAL SYSTEMS}

The intent of this section is to briefly describe some fundamental principles in the formal representation of causality. The most important feature of this presentation is that this mathematical representation is extended from the usual linear algebraic form to some new principles in diagrammatic nomography.

\section{Causal Assumptions}

The formal logic behind broad definitions of causality can be described by the three fundamental assumptions of all causal statements: (1) Functional-The expected values of effect events are conditional upon given values of cause events with some nonzero probability. (2) Chronological-Cause events must precede effect events in some dimension of time for any causal relationship to obtain. (3) Bounded-Causality is only relative to some completely defined conceptual framework in which both probability and chronology range only over a specified class of events.

The first assumption of functional form, a general "principle of covariation" (Kelley, 1973), can be more precisely described by the mathematical form of general multivariable linear regression:

$$
P=\exp (E \mid C=\mathrm{G})
$$

or

$$
E=C D_{\mathrm{ec}}{ }^{\mathrm{t}}+R \text {, }
$$

where exp = expected value operator (over $\mathrm{N}$ subjects), $C=$ a matrix of cause variables, $E=$ a matrix of effect variables, $\mathrm{G}=\mathrm{a}$ matrix of given values, $P=$ a matrix of predicted values, $D=$ a matrix of regression coefficients, and $R=$ a matrix of residual variables.

The critical assumption here is that causes and effects can be considered in Galtonian probabilistic terms rather than in Humean deterministic fashion. Many western philosophers (e.g., Suppes, 1970) claim that this is an essential feature of any realistic set of causal propositions. However, MacWhinney (1978, p. 406) usefully points out that "functions and associations serve to map one set of values onto another but say little about the action performed ... in achieving the mapping." The functional basis, then, is only considered to be reflective of a level of reductionism that proves most useful for the task at hand.

This representation also requires an additional assumption of linearity in relationships. Linearity permits all relations to be stated in terms of additive and proportional magnitudes, but it does not rule out nonlinear relations (e.g., Cohen \& Cohen, 1975). In this way, the causal composition of covariation may also be stated as follows: "A given change in the cause variables always involves a certain constant change in the values of the effect variables" (Wright, 1921).

The only mathematical addendum required by the chronological assumption is that $D_{\mathrm{ab}}=0$ if some variable $a$ precedes some variable $b$ in time. This is important because there is no inherent mathematical necessity to adhere to this rule (Wright, 1934). These chronological mathematics also provide a natural extension for modeling complete causal systems. In cases in which variables are all observed at a single discrete time interval, a microchronologic cause-effect assumption must be invoked. Due to the nature of this fixed "time slice," the functional $D$ relations are said to refer to synchronic, steady state system structure. In other cases, in which variables are observed at many specified discrete intervals on a defined time continuum, a macrochronologic cause-effect assumption is invoked. Functional $D$ s refer to kinematic, dynamic state system processes. Thus, differences between structure and process interpretations are defined extramathematically by observational design (Rozeboom, Note 2).

The boundary assumption places strong requirements on the theoretical model and its sampling bases. Statistically speaking, the expectation operator (Equation 1) places boundaries of independence and homogeneity of subjects within a specified group. More important here, however, is the metrical assumption that all variables that have any nonzero cause or effect in the complete network of relations must be included in the system. This requires all "spurious" or "third-variable" causes (Wright, 1921) to be specifically considered. It also permits representations that are highly complex, such as causes cancelling out each other. These boundary assumptions are most severe and perhaps the most open to criticism.

This causal definition still does not fully specify all essentials of system mathematics. Without a doubt, this is partially due to the lack of explicit mathematical treatments in the general systems literature. For example, the classic definition of a system is "a collection of entities and sets of relations among them" (Rapoport, 1972, p. 45). One useful systems conception arising from the psychological literature was offered by Cattell $(1966,1978)$. First, he globally described a "reticule of interlocking influences" as the "most general scientific model." Unlike many others, however, he tried to link these ideas with the modern mathematics of factor analysis. A formal extrapolation of Cattell's ideas will now be described. 


\section{RAM Nomography}

The essential basis of RAM (McArdle, 1979, Note 1) is that the formal features of all causal systems action can be completely described using only a few necessary theoretical distinctions. These ideas tum out to be completely isomorphic with specialized pathdiagram/linear-flowgraph representation that, due to their functional-graphical form, are referred to as nomographs. The necessary distinctions may be divided into two categories. (1) $R A M$ variables-Any variable ( $V$; $t$ in number) for any subject ( $\mathrm{S} ; \mathrm{N}$ in number) is defined using one of two nomographical elements: (a) manifest $(\mathrm{M} ; \mathrm{j}$ in number)-a variable that is an observed (or assigned) attribute and drawn as a square, and (b) latent ( $L ; k$ in number)-a variable that is postulated as a hypothetical attribute (italic notation in Figure 1) and drawn as a circle. (2) $R A M$ relations-The only possible forms of relations among variables can also be described using two additional nomographic elements: (a) Directed (D)-Causal relations between any variables may be described in terms of the regression coefficients in

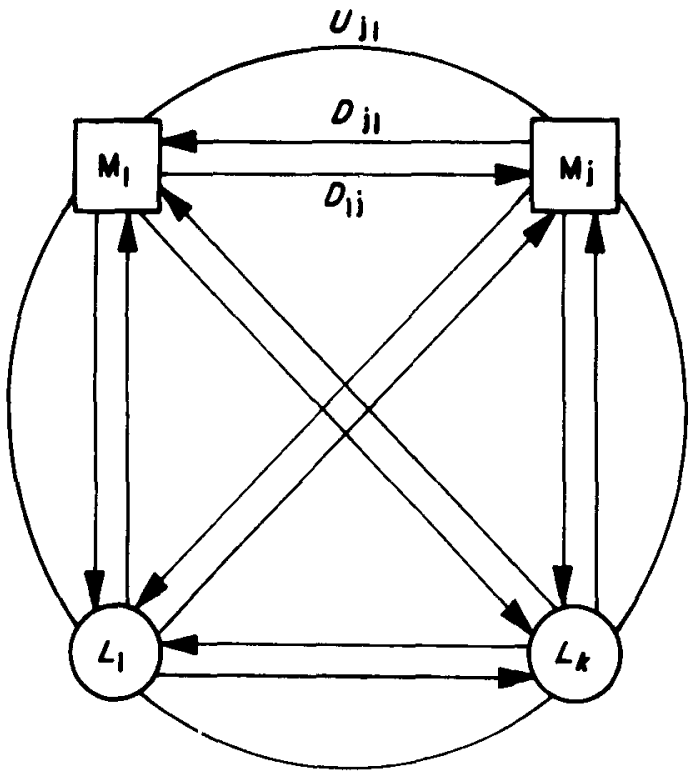

KEY

\section{Voriables \\ $M=$ Monifest Squores $(1, \ldots j)$ \\ $L=$ Lotent Circles $(1, \ldots h)$ \\ Relotions \\ $D=$ Directed Arrows \\ $U=$ Undirected Lines \\ Structure

$$
C=\mathrm{J}(I-D)_{-1} U(I-D)^{-1} \mathrm{~J}
$$

Figure 1. Reticular analy tic modeling (RAM) theory.
Equation 1. Each one-directional relation is written as a one-headed arrow pointing from the cause variable $c$ and toward the effect variable $e$, with the associated $d_{\text {ec }}$ coefficient usually placed on a straight path. (b) Undirected $(U)$-Noncausal relations between any variables may be simply described as allowed or permitted. In the usual regression equation, these include variances and correlations of residuals and of exogenous (independent) variables. These symmetric undirected relations are written as no-headed lines between variables $i$ and $j$ with the associated $u_{\mathrm{ij}}$ value usually placed on a curved path.

A complete nomographical representation can be presented as in Figure 1. This shows that there are only four distinct graphic elements: All variables are either squares or circles, and all relations are either one-headed arrows or no-headed lines. These four pictorial elements may be considered the four nomographic primitives of RAM theory.

The diagram illustrates that all possible causal relations among both $\mathrm{M}$ and $L$ can be drawn. To retain clarity, not all of these possibilities are labeled. Some directed causal relations are represented, for example, by the one-headed arrow between cause $M_{1}$ and effect $\mathrm{M}_{\mathrm{j}}$, with this relation given as $D_{\mathrm{j} 1}$. The opposite causal interpretation is given to $D_{1 \mathrm{j}}$. Historically, it is interesting to note that this representation of $D$ is equivalent to Cattell's (1966) original reticular diagram. While all variables are drawn as $\mathrm{M}$ or $L$, no special residual variables are necessary. The characteristics of these residuals may simply be considered the undirected relations of $V$ as drawn here. For instance, the $U_{1 \mathrm{j}}$ is simply the undirected relation between $\mathbf{M}_{1}$ and $\mathbf{M}_{\mathbf{j}}$. While all possible $U$ relations are not drawn (e.g., $U_{1 k}$ between $\mathrm{M}_{1}$ and $L_{k}$ ), all are conceptually possible.

\section{RAM Algebra}

For many practical reasons, it is important to present RAM nomography in a convenient matrix algebra formulation. However, because most of the mathematical principles described here are based on the work of Jöreskog and Sörbom (1978) and McDonald (1979), a complete description of RAM algebra will not be provided (cf. McArdle, Note 1). RAM represents a general multivariable regression system that is algebraically developed for the gth $(g=1, \ldots, G)$ group by writing the moment structure form as

$$
W=\exp \left(\mathrm{M}^{\mathrm{t}} \mathrm{M}\right)=\mathrm{J}(\mathrm{I}-D)^{-1} U(\mathrm{I}-D)^{-\mathfrak{t} \mathbf{J}^{\mathrm{t}}},
$$

where $W=$ general moment matrix (size $\mathrm{j}$ by $\mathrm{j}$ ) including means, variances, and correlations (the $C=$ covariance matrix equation is equivalent; see Figure 1), $M=$ manifest variable (data) matrix ( $N$ by $j$ ), $J=$ index matrix $(j$ by $t$ ) of zeros with unit manifest indexes, $\mathrm{I}=$ identity matrix ( $t$ by $t$ ), $D=$ directed matrix ( $t$ by $t$ ) of oneheaded relations, and $U=$ undirected matrix ( $t$ by $t$ ) of no-headed relations. 
These matrix computations include only subtraction, inversion, transposition, and multiplication. The most important feature of this RAM equation is that once values are given to $D$ and $U$ parameters, the matrix computations transform these into population parameters of $W$ for the means, variances, and correlations. In this way the general RAM equation can be used to transform a hypothetical conception of causality into a set of data that is strictly consistent with these ideas. Unfortunately, the important question concerning the specific values to assign to parameters is not also a mechanical procedure. These algebraic values may be most easily examined by their direct relationship to the nomography.

\section{Nomography to Algebra}

The conceptual simplicity of the RAM algebra resides in the fact that it is completely isomorphic with the nomography. A specific stepwise translation scheme can be presented as follows. (1) Nomographic drawing-Define and draw a substantive theory in terms of necessary RAM specifications: (a) variables-the number of manifest squares $(\mathrm{j})$ and latent circles $(k)$ (aligned as the $t$ variables in matrix $V$ ), and (b) relations-the particular values of the parameters that are causally one-headed $(D)$ and noncausally no-headed $(U)$. (2) Obtain index-Create $J$ as a null rectangular matrix with the $\mathrm{j}$ manifest variables $\mathrm{M}$ as rows and all $t$ variables $V$ as columns. Simply assign a unit value to the row and column intersection that refers to the same manifest variable. (3) Obtain directed-Create $D$ as a null square matrix with all $t$ variables as indices of both the rows and columns. Place the one-headed pathways from the nomograph as parameters with column variable input to row variable. (4) Obtain undirected-Create $U$ as a null square and symmetric matrix with all variables as indices of both the rows and columns. Place the no-headed lines from the nomograph as parameters in the main diagonal and lower (or upper) triangle sector. It should also be clear that an algebra-to-nomography translation requires merely a reversal within each of these steps.

The $D$ and $U$ parameter values may be considered akin to valves in a hydraulic system; the value describes the valve type and position. For instance, a specified value of zero can constitute a "closed" valve, and any nonzero value can be considered "open." The size of this coefficient is reflective of the strength of the relation (e.g., high or low), and the sign of the value describes the control function of the valve (e.g., increasing or decreasing). As with hydraulic systems, the effect that a given valve may have in a given system may be complex. For instance, two systems with the same valves can produce different output (e.g., open to different strengths), or two systems with completely different valve settings may produce identical output (e.g., alternative equilibrium). A wide variety of valve configurations are possible even for very simple systems.

The major point is that the variety and effect of each parameter can be defined only by reference to a full set of parameters, termed the structural model (Bock \& Bargmann, 1966). This term is used because a collection of parameter settings provides a specified set of limitations on the structure of elements of the population moments (and is not reflective of the structure-process distinction). The value of any individual parameter, therefore, cannot be specified outside a prescribed contextual boundary. Due to these characteristics, the issues and problems can quickly become complex and confusing.

\section{EXAMPLES OF CAUSAL SYSTEM SPECIFICATION}

This section provides a formal exercise in the fundamental principles involved in the allocation and interpretation of causal parameters.

\section{Regression Analysis}

The basic example of multiple regression, presented here as Figure 2.1 , provides a useful starting point. In the early development of path analysis (Wright, 1921, 1934), all manifest variables were considered only

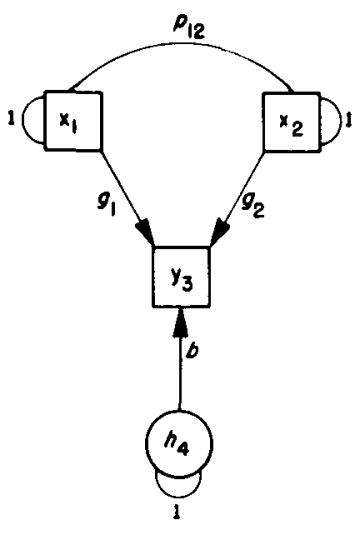

1. Multiple Regression

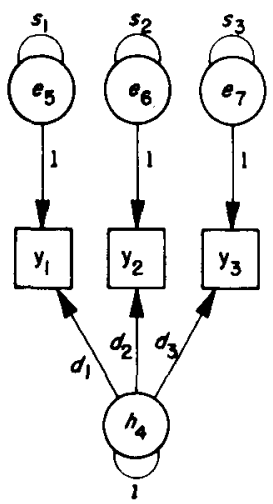

3. Foctor Analysis

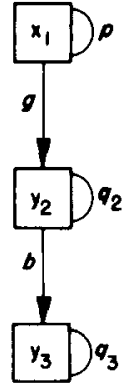

2. Chain Simplex
Figure 2. Specific examples of RAM nomography. 
in standardized form, and model parameters were developed only for a correlation matrix (now, as usual, symbolized by $R$ ). Thus, the multiple-regression model applied to a set of correlations is a good starting point.

The causal interpretation of this model proves quite simple: Observed $\mathrm{x}_{1}$ and $\mathrm{x}_{2}$, as well as hypothetical $h_{4}$, cause observed effect $y_{3}$. The undirect relations merely state that all cause variables are standardized and that $x_{1}$ and $x_{2}$ causes are allowed to be correlated. One important model restriction is the lack of any relation between $h_{4}$ and the other causes, $x_{1}$ and $x_{2}$. Now that all relationships are explicit, this model can be written algebraically as

$$
\mathrm{y}_{3}=\mathrm{x}_{1} g_{1}+\mathrm{x}_{2} g_{2}+h_{4} b,
$$

with $\exp \left(\mathrm{x}_{1}{ }^{\mathrm{t}} \mathrm{x}_{1}\right)=\exp \left(\mathrm{x}_{2}{ }^{\mathrm{t}} \mathrm{x}_{2}\right)=\exp \left(h_{4} \mathrm{t}_{4}\right)=1$ and $\exp \left(\mathrm{x}_{1}{ }^{\mathrm{t}} \mathrm{x}_{2}\right)=p_{12}$.

It is useful to write some general rules for the reconstruction of the hypothesized correlation matrix elements from the nomograph parameters. These path-flow rules, originally developed by Wright $(1921,1934$; but see $\mathrm{Li}, 1975$, pp. 161-165), illustrate the control feature of each nomograph parameter in a particular correlation $r_{\mathrm{ij}}$. These can be obtained by the following travelogue through the nomograph:

(1) Correlational summation-The correlation of variable $i$ and variable $j$ is the sum of all distinct nomographic pathways from $i$ to $j$. Note here that $i$ and $j$ could be the same variable, and a variance term (unit coefficient of determination) would result. (2) Path products-A nomographic pathway from $i$ to $j$ is the product of all path parameters traveled along (on the nomograph) in getting from $i$ to $j$. This pathway is distinct if no other travel is identical from start to finish. (3) Backward start-A pathway usually begins by moving from $i$ along a path whose arrowhead points backward to $i$. Travel along an undirected path may also be considered as a starting point. (4) Forward turn-A single turning point toward forward arrowhead travel is permitted in each pathway by traveling through an undirected path. This is not necessary if the $j$ destination has been reached.

Perhaps the most important restrictions are those that limit the structure of the dependent variable $y_{3}$ correlations to be a linear function of the other model parameters. Using these nomograph travel rules, the elements of the hypothesized $y_{3}$ correlations may be written as

$r_{13}=\exp \left(\mathrm{x}_{1} \mathrm{y}_{3}\right)=g_{1}+g_{2} p_{12}$

$r_{23}=\exp \left(\mathrm{x}_{2}{ }^{\mathrm{t}} \mathrm{y}_{3}\right)=g_{2}+g_{1} p_{12}$

$r_{33}=\exp \left(\mathrm{y}_{3}{ }^{\mathrm{t}} \mathrm{y}_{3}\right)=1=g_{1}{ }^{2}+g_{2}{ }^{2}+2 g_{1} g_{2} p_{12}+b^{2}$.

Here the correlations are complex functions of the model parameters. Therefore, changes in any parameters can alter the correlations in nonobvious ways.
The utility of the RAM equation in this regard is that, once parameter values are given, the RAM equation performs all of these operations in one stroke. Using this model as the example and RAM notation with asterisks, the stepwise nomography-translation scheme begins by setting up the full matrix of all variables as

$$
\underset{(\mathrm{N} \text { by } 4)}{V^{*}}=\left[\begin{array}{llll}
\mathrm{x}_{1} & \mathrm{x}_{2} & \mathrm{y}_{3} & h_{4}
\end{array}\right] .
$$

The manifest index matrix is created with the $M$ variables as rows and all $V^{*}$ variables as columns. Somewhat mechanically, a unit value is then placed at the intersection of the row and column variables:

$$
\begin{aligned}
& \begin{array}{llll}
\mathrm{x}_{1} & \mathrm{x}_{2} & \mathrm{y}_{3} & h_{4}
\end{array} \\
& \mathrm{~J}^{*}=\mathrm{x}_{1}\left[\begin{array}{llll}
1 & 0 & 0 & 0
\end{array}\right]=\left[\begin{array}{lll}
\mathrm{I} & : & 0
\end{array}\right] \\
& \text { (3 by } \left.4 \text { ) } \quad x_{2} \mid \begin{array}{llll}
0 & 1 & 0 & 0
\end{array}\right] \quad \text { (3 by } 3 \text { ) (3 by } 1 \text { ) } \\
& y_{3}\left[\begin{array}{llll}
0 & 0 & 1 & 0
\end{array}\right]
\end{aligned}
$$

The directed matrix is specified by setting up a $V^{*}$ by $V^{*}$ square supermatrix and entering the oneheaded arrows as parameters in which the column variable causes the row variable:

$\begin{array}{cl}D^{*} \\ (4 \text { by } 4) & \mathrm{x}_{1} \\ & \mathrm{x}_{2} \\ \mathrm{y}_{2} \\ h_{4}\end{array}\left[\begin{array}{llll}0 & 0 & 0 & 0 \\ 0 & 0 & 0 & 0 \\ g_{1} & g_{2} & 0 & b \\ 0 & 0 & 0 & 0\end{array}\right]$

The undirected matrix is set up as a $V^{*}$ by $V^{*}$ lower triangular symmetric matrix in which the no-headed lines are entered at row and column intersections:

$\begin{array}{cl}U^{*} \\ (4 \text { by } 4) & \mathrm{x}_{1} \\ \mathrm{x}_{2} & \mathrm{y}_{3} \\ h_{4}\end{array}\left[\begin{array}{llll}\mathrm{x}_{1} & \mathrm{x}_{2} & \mathrm{y}_{3} & h_{4} \\ p_{12} & 1 & & \\ 0 & 0 & 0 & \\ 0 & 0 & 0 & 1\end{array}\right]$

As a concrete example of this model, let $g_{1}=.5$, $g_{2}=.4, b=.6$, and $p_{12}=.575$. Upon substitution of these parameters into Equation 2 (or Equation 4), this model creates

$$
W^{*}=R=\left[\begin{array}{rrr}
1.000 & & \text { sym } \\
.575 & 1.000 & \\
.730 & .688 & 1.000
\end{array}\right]
$$

The necessity to create a correlation matrix places additional mathematical constraints on the possible parameters: The main diagonals must all be $1 \mathrm{~s}$, and the off-diagonals must range between +1 and -1 . In this example, $g_{1}, g_{2}$, and $b$ were first specified. Then $p_{12}$ was calculated under the constraint that $r_{33}=1$. Of course, there are many alternative ways to create models 
under the identical constraints, and more general principles will be given later. The important point is that once values are given to the causal parameters, a set of population data may be directly produced from the RAM equation.

A slightly more complex path model is presented in Figure 2.2. In this figure, a few new pictorial conceptions are introduced. First, a full covariance specification $\left(W^{*}=C\right)$ is presented, in which variables are not scaled in any simple fashion (e.g., standardized). Second, the $q$ are not considered as the special regression coefficient of an unobserved $h$ variable, but, equivalently, they are seen as variance that is specific to the criterion variable. In this type of compact-path nomograph, the unique latent variable is not drawn, but its regression effect is treated as disturbance variance. In general, the undirected relation from any variable back to itself is the unique variance of that variable. If there are no other variables causing it, this undirected relation is interpreted as its total variance.

Some new causal features are also introduced by this diagram. Figure 2.2 represents a model of a "causal chain" (Wold, 1954; Wright, 1921) or "simplex" (Guttman, 1954); the $x_{1}$ causes $y_{2}$, which in turn causes $\mathrm{y}_{3}$. The $p$ is the variance of $\mathrm{x}_{1}$, and the $q_{2}$ and $q_{3}$ represent disturbances from the previous cause. If these variables were measurements at three consecutive times, the $g$ and $b$ could represent first-order autoregressive dynamic process coefficients. The temporal logic of causal propositions would require that the dynamic effect of $\mathrm{y}_{3}$ causing $\mathrm{y}_{2}$ (i.e., $b_{23}$ ) equal zero, but there are no such inherent mathematical restrictions.

Due to the pictorial absence of explicit disturbances, this model can be compactly specified as $t^{*}=3$, and

$$
\begin{gathered}
V^{*}=\left[\begin{array}{lll}
\mathrm{x}_{1} & \mathrm{y}_{2} & \mathrm{y}_{3}
\end{array}\right] \\
\mathrm{J}^{*}=\begin{array}{c}
\mathrm{I} \\
(3 \text { by } 3)
\end{array} \\
D^{*}=\quad\left[\begin{array}{lll}
0 & 0 & 0 \\
g & 0 & 0 \\
0 & b & 0
\end{array}\right] \\
U^{*}=\left[\begin{array}{lll}
p & \mathrm{sym} \\
0 & q_{2} & \\
0 & 0 & q_{3}
\end{array}\right]=\operatorname{diag}\left[\begin{array}{lll}
p & q_{2} & q_{3}
\end{array}\right],
\end{gathered}
$$

where diag = matrix operator signifying diagonal elements only.

\section{Factor Analysis}

Some novel RAM views of latent variables are presented by the basic model of common factor analysis (cf. Thurstone, 1947) given in Figure 2.3. In this model the three manifest $y_{i}$ are caused by one hypothetical common factor $h_{4}$, with pattern loadings $d_{\mathrm{i}}$ and unit variance. Three specific factors, $e_{5}, e_{6}$, and $e_{7}$, with variance $s_{\mathrm{j}}$ (and unit regressions) are also postulated as causes. This causal model has been written without the unique paths as parameters because it is useful to see that other parameterizations (e.g., in which the $e_{j} \mathrm{~s}$ are given unit variance and regression paths equal to the square root of $s_{j}$ ) represent exactly equivalent alternatives.

This model can be translated directly into RAM equations of the following form:

$$
\begin{aligned}
& V^{*}=\left[\begin{array}{lllllll}
\mathrm{y}_{1} & \mathrm{y}_{2} & \mathrm{y}_{3} & h_{4} & e_{5} & e_{6} & e_{7}
\end{array}\right] \\
& \mathrm{J}^{*}=\underset{(3 \text { by } 4)}{\left[\begin{array}{cc}
\mathrm{I} & 0
\end{array}\right]} \\
& D^{*}=\left[\begin{array}{lllllll}
0 & 0 & 0 & d_{1} & 1 & 0 & 0 \\
0 & 0 & 0 & d_{2} & 0 & 1 & 0 \\
0 & 0 & 0 & d_{3} & 0 & 0 & 1 \\
0 & 0 & 0 & 0 & 0 & 0 & 0 \\
0 & 0 & 0 & 0 & 0 & 0 & 0 \\
0 & 0 & 0 & 0 & 0 & 0 & 0 \\
0 & 0 & 0 & 0 & 0 & 0 & 0
\end{array}\right] \\
& U^{*}=\operatorname{diag}\left[\begin{array}{lllllll}
0 & 0 & 0 & 1 & s_{1} & s_{2} & s_{3}
\end{array}\right] \text {. }
\end{aligned}
$$

A concrete example of this model can be written with $d_{1}=.6, d_{2}=.7, d_{3}=.8, s_{1}=.64, s_{2}=.51$, and $s_{3}=.36$. Upon substitution into Equation 2, this yields

$$
W^{*}=R=\left[\begin{array}{rrr}
1.00 & & \text { sym } \\
.42 & 1.00 & \\
.48 & .56 & 1.00
\end{array}\right]
$$

A more complex causal structure is postulated by Figure 2.4. In this model the $y_{1}$ and $y_{2}$ are caused by latent $h_{4}$ and the $\mathrm{y}_{2}$ and $\mathrm{y}_{3}$ are caused by latent $h_{5}$. The overlap of factorial causality in $y_{2}$ represents a pileotropic/alternative-mechanism view of factorial complexity (Horn, 1978). Another major feature is that $h_{4}$ and $h_{5}$ are also caused by a higher order factor $c_{6}$. All variables are written with an undirected unique variance that is interpreted as "disturbance" variance, except the highest order, $c_{6}$. This entire model represents a simple version of hierarchical factor causal influence system (Cattell, 1966, 1978) that may be specified by using the same basic translation principles. The only difference in the matrix equations is that they typically become larger and sparser as more variables are introduced into the nomograph.

\section{Interdependent Systems}

Figure 3 illustrates additional combinations of these ideas. In Figure 3.1 a crucial path model for two sets of variables with one common latent variable is presented. Here, $h_{5}$ is depicted as a composite effect of $x_{1}$ and $x_{2}$ 


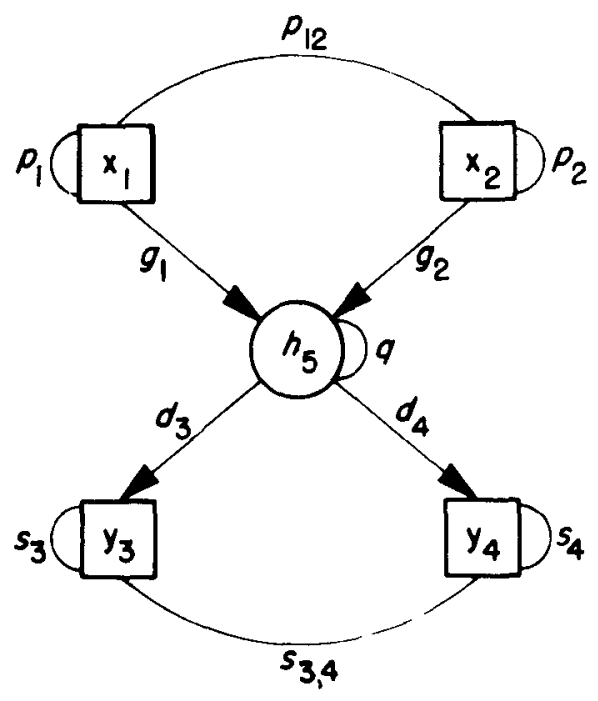

1. Multiple Indicators/Multiple Couses

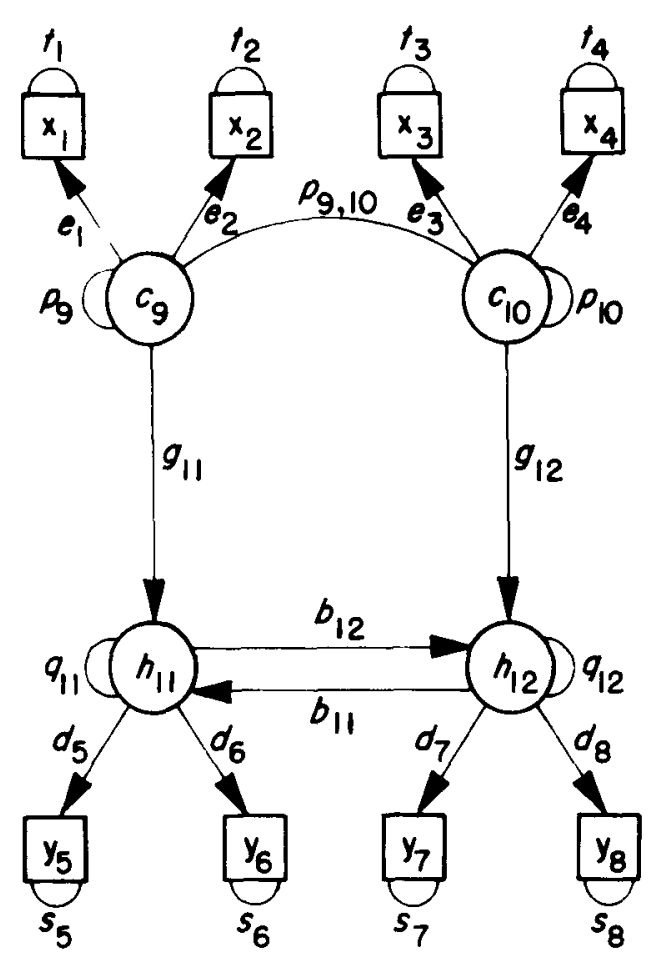

2. Interbottery System

Figure 3. Interdependent systems nomography.

but also as a cause of $\mathrm{y}_{3}$ and $\mathrm{y}_{4}$. The $s_{34}$ represents the partial correlation of $\mathrm{y}_{3}$ and $\mathrm{y}_{4}$ with $h_{5}$ partialled out. This multiple-causes/multiple-effects model shows that manifest variables can be direct causes of latent variables and vice versa. This model bears a strong resemblance to the analytic models described as "MIMIC" (Jöreskog \& Goldberger, 1975) and "canonical redundancy" (van den Wollenberg, 1977).

Figure 3.2 presents a more complex model of two manifest batteries $x_{i}$ and $y_{j}$. The four $x_{i}$ variables are hypothesized to be manifest outputs of two nonoverlapping correlated (oblique) latent common factors, $c_{9}$ and $c_{10}$. The $y_{j}$ are considered outputs of two nonoverlapping common factors, $h_{11}$ and $h_{12}$, respectively. In this model all interbattery relations among manifest variables exist in direct causal fashion only at the latent common factor level. Factors $c$ are allowed covariance $p$ and are direct causes of $h$. If these $\mathrm{x}_{\mathrm{i}}$ were considered to be measured at Time 1 and $y_{j}$ at Time 2, the $g$ could represent dynamic latent process coefficients.

The interrelationship of the latent $h$ illustrates direct feedback loops. This type of causal construction (nonintuitively termed "recursive" after the equational form of $D$ ) must be causally interpreted as " $h_{11}$ causes $h_{12}$ and $h_{12}$ causes $h_{11}$." One way to come to grips with the necessary chronological assumption of causality in this model is to suggest that the observation has captured a multiple sequence of causes and effects. That is, the observational time slice is too wide to permit discrete separation of true chronological cause and effect. The flowpath description of this relationship is complicated by the fact that travel along the inner loop must be considered as an infinite cycle. This can be written in mathematical form as an inverse function of the parameters that, in general, is given as Equation 2.

\section{Mean Parameters}

There are many ways to represent the means in the traditional ANOVA fashion. In one popular approach the $x_{i}$ variables are created to represent hypothetical relations between groups (e.g., contrast, dummy, effectcoded group differences). Subjects from all groups are then collapsed to form a single covariance matrix including both independent and dependent variables.

Consider Figure 2.1 to represent the simple example of a three-group univariate ANOVA design. The $\mathrm{x}_{1}$ and $x_{2}$ can represent the breakdown of the independent group variable into its two degrees of freedom by using "effects" coding (Cohen \& Cohen, 1975): Let (1) $\mathrm{x}_{1}=1$ if the subject is a member of Group $1, x_{1}=-1$ if the subject is in Group 3, and $x_{1}=0$ if the subject is in Group 2, and (2) $x_{2}=1$ if the subject is a member of Group 2, $x_{2}=-1$ if the subject is in Group 3, and $\mathrm{x}_{2}=0$ if the subject is in Group 1. Of course, $\mathrm{y}_{3}$ represents the dependent variable of interest. The latent $h_{4}$ represents all other variables, uncorrelated with 
group assignment, that cause $y_{3}$ variation. The model parameters are now all interpretable in strict ANOVA fashion. The $p_{12}$ merely reflects the fixed design characteristic of group sizes (e.g., with equal group sizes, $p_{12}=.5$ ). Most important, $g_{1}$ and $g_{2}$ are interpreted as the change in $y_{3}$ that is caused by assignment to a specific group (i.e., between groups). Finally, $b$ represents all variation in $y_{3}$ that remains unexplained (i.e., residual) by group assignment.

Due to the clarity of other presentations on this topic (e.g., Cohen \& Cohen, 1975), a more extensive discussion of this logic will not be presented here. It is important now only to appreciate the greater flexibility of this approach compared with traditional ANOVA. For instance, Figure 3.1 can be considered as an extension of this logic to multivariate (MANOVA) form. This figure portrays the causal effects of independent variables $\mathrm{x}_{1}$ and $\mathrm{x}_{2}$ on $h_{5}$ rather than on $\mathrm{y}_{3}$ and/or on $y_{4}$ directly. The same variety of modeling would permit the representation of group differences on latent common factors (i.e., $s_{34}=0$ ). Many other "mixed models" are now also possible (McArdle, 1978).

Sörbom $(1974,1978)$ has introduced an important alternative view of differential group intercept parameters. In the nomographic approach, a new feature must be introduced to the diagram given in Figure 4: Another square is used to denote a constant $\mathrm{k}$. This $\mathrm{k}$ is considered to be the special variety of manifest variable (handled here as an $\mathrm{x}$ variable) that has exactly the same score for each subject, usually given as one. This implies zero variation and zero correlation of $k$ with all other variables. The importance of the $k$ variable is that its direct relation with the other variables represents the usual location means (written with bars overhead).

The output data now consist of means $(\bar{M})$ and covariances $(C)$, with the addition of $\mathrm{k}$ as a manifest variable. Thus, the population is described by a constant augmented-moment structure:

$$
\begin{aligned}
W^{*}= & C^{*}+\bar{M}^{* \mathrm{t}} \bar{M}^{*} \\
& =\left[\begin{array}{llr}
1 & \\
\bar{y}_{2} & \left(c_{22}+\bar{y}_{2}{ }^{2}\right) & \mathrm{sym} \\
\bar{y}_{3} & \left(c_{32}+\bar{y}_{3} \bar{y}_{2}\right)\left(c_{33}+\bar{y}_{3}{ }^{2}\right)
\end{array}\right]
\end{aligned}
$$

One simple example of this parameterization is given in Figure 4.1. This model represents a simple regression model on the complete moment structure for a single variable measured at two time points. This is usually termed a "dependent-samples t-test design." As always, mean parameters must be considered in relation to the rest of the model, so the criterion mean is now a path function of the other regression parameters, namely, $\bar{y}_{2}=\bar{g}_{3}+\bar{g}_{2} b$. This illustrates the novel RAM principle that the original path correlation reconstruction rules can also apply to the full augmented-moment structure.

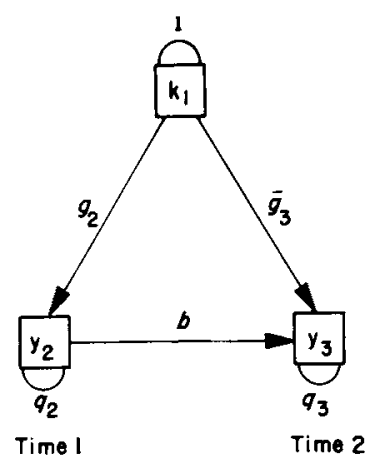

1. Dependent $/$-Design

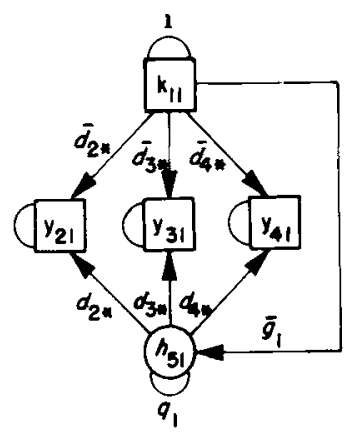

Group 1

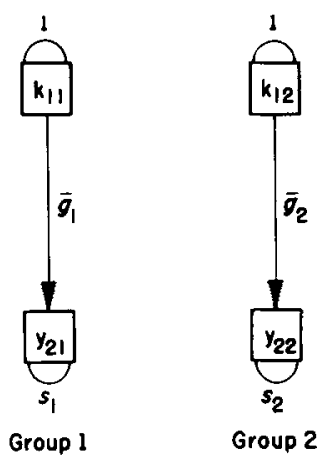

2 Independent $t$-Design

\section{Lotent Voriable Group Means}

Figure 4. Nomographical representations of mean parameters.

The causal features of this model are somewhat unusual. While it is easy to state that $y_{2}$ causes $y_{3}$, it seems most awkward to say that a constant $k_{1}$ causes both of them. By invoking mechanistic principles (Bock, 1975), however, a reasonable dialogue can be sustained. In these terms the mean vector $\bar{y}_{1}=\bar{g}_{2}$ is termed the centroid. This represents momentum from a zero starting point. The covariances are now termed inertia for the constancy of their momentum from the centroid. It follows that $\bar{g}_{3}$ represents centroid shift from a starting point that is determined by the inertial proportion of the Time 1 location; $\bar{g}_{3}$ is unpredicted location change due to observational time difference. Although means are not usually considered in this fashion, it seems quite consistent to do so here.

Figure 4.2 offers the possibilities of considering several independent groups simultaneously (the last subscript indicates group). This simple model for two groups on one variable permits considerations of the traditional "independent-groups t-test design" and also includes differential group variances $s_{\mathbf{j}}$. One advantage of this formulation is that the means are represented in the more "natural" magnitudes of means rather than "effect sizes." It also seems that multiple groups permit 
a more convenient way to study Group by Variable interactions (Reichardt, Note 3). There are many ways that these ANOVA formulations can be extended into structured-mean representations (for more detailed examples, see Horn \& McArdle, 1978; McArdle, 1978).

Another important use of this logic is in its easy extension to latent variable means. The comparison of factor means across multiple groups (Sörbom, 1974) is depicted in Figure 4.3. Once again, the data to be analyzed are in the form of the mean augmented-moment matrix. This includes four manifest variables $\left(k_{1}, y_{2}\right.$, $\left.y_{3}, y_{4}\right)$ for each of the two groups. The covariance structure proposed is that of a single common factor $h_{5}$, drawn as a compact path (cf. Figure 2.3). In this model, however, all factor-loading parameters, $d_{\mathrm{i}^{*}}$, are further hypothesized to be equal over groups (i.e., invariance over groups indicated by an asterisk). Covariance differences between groups can now only arise from the latent variable variances, $s_{\mathrm{i}}$ and $q_{\mathrm{j}}$. The mean structure suggests that there is some grand mean $\left(\bar{d}_{\mathrm{i}^{*}}\right)$ for each variable, which is also set to be equal over groups. This parameterization permits control over all mean differences between groups only at the latent factor mean $\vec{g}_{j}$ level. This type of model might be considered a practical mixture of the experimental and correlational paradigms (Cattell, 1966).

\section{ON-LINE SIMULATION OF CAUSAL SYSTEMS}

There exist many possibilities for utilizing the RAM conception in the task of simulation. One practical scientific model and its algorithmic solution are discussed in this section.

\section{RAM Plasmodes}

The simulation proposed here is limited to the form of a plasmode, which, in the words of Cattell and Jaspers (1967, p. 3), can be defined as follows: "A numerical scientific example, preferably derived from data in a physical model, in which the relations generating measures are controlled, and known to fit an explicit theoretical model. It derives from plasma $(=$ form) and mode (= measurement), thus aptly designating an act of measurements which fit a particular form or structure .... The best kind of plasmode for most purposes will be completely 'realistic' by having suitable sampling and experimental errors incorporated with the measurements ... . Numerical values representative of typical ranges ... [and] maximally simulate all that one would encounter in applying research methods and analyses to real data."

The major utility of RAM nomography in this regard is that any nomograph proposes a limiting structure on the hypothesized population moments. When the $D$ arrows and $U$ lines are given explicit values, the population-moment $W$ s (means, variances, and correlations) are explicitly defined by Equation 2 . Therefore, in order to simulate data from any hypothesized causal systems model, one need only specify the population mechanism, calculate the proposed moment structure, and specify the generation of sample-moments $\mathrm{W}$ data from the given population. Other transformations for applied realism can then be applied to these data.

It is important to consider other alternatives that suggest that this method is only one possible plasmode. For example, both Cattell and Jaspers (1967) and Wackwitz and Horn (1971) generate simulation data based on the specified characteristics of all latent variables. When this is done, they proceed to transform these into manifest scores by a mathematical model. While the computations are usually increased, some conceptual features of the process are clarified. Baltes, Nesselroade, and Cornelius (1978) go one step further by attempting to simulate each individual score going through a transformation process. This, of course, is a movement toward deterministic/discrete-event logic and practically eliminates the difficulties in specifying any underlying statistical distribution mechanism (cf. Chambers, 1970). All of these procedures have useful properties for specific types of problems, and they are not inconsistent with the goals of the RAM plasmode conception.

\section{Computer Algorithms}

The actual process suggested here begins by using the numerical solutions that exist for statistical sampling under the general assumptions of multivariate normality (see McArdle, 1976). In order to randomly generate multivariate data with a prescribed population mean and covariance structure, the following procedure is typically used:

$$
\begin{aligned}
& C=A A^{\mathrm{t}} \\
& \mathrm{Z} \Rightarrow \mathrm{N}(0, \mathrm{I}) \\
& \mathrm{M}=\mathrm{Z} A^{\mathrm{t}}+\bar{M} \Rightarrow \mathrm{N}(\bar{M}, C),
\end{aligned}
$$

where $Z=$ normally distributed $\left[\Rightarrow \mathrm{N}\left({ }^{*}\right)\right]$ matrix (N by j) of raw scores with mean vector 0 and covariance matrix $\mathrm{I}$, and $A=$ transformation matrix (j by j) with null upper triangle.

The $A$ matrix is first calculated using any square-root factoring procedure (also termed Cholesky or Crout). This procedure requires the initial generation of random data matrix $\mathrm{Z}$, which usually begins by calculating a matrix of uniform random deviates (e.g., Marsaglia, 1976) transformed columnwise to normality (e.g., Ahrens \& Dieter, 1972). The rest of the process involves the necessary matrix - multiplication (e.g., Lehman, 1977). One generally good and available algorithm for this procedure is the GGNRM routine in the IMSL (1979) package.

Other procedures simulate the moments without 
simulating all of the raw data. Odell and Fieveson (1966) show how $C$ may be simulated directly for any sample size by generating only $\mathrm{j}$ chi-square variates (main diagonals) and $j(j-1) / 2$ normal variates (off-diagonals). Chambers (1970) further attempts to show how the means may be simulated directly by generating only $j^{2}$ normal variables. These have the greatest practical utility in large-sample studies (Montanelli, 1975).

One important feature of these mathematics is the requirement that $C$ be positive-definite (Gramian) due to the factoring in Equation 14. This can be assured under RAM theory only under some additional constraints. For example, while arbitrary values in $U$ may not produce Gramian $C$, this condition is typically met with positive and diagonal $U$. Conceptually, this suggests that the hypothesized causal model should be created from directed $D$ relations as much as possible. Therefore, causally directed arrows should be used whenever possible under the invertible $D$ side conditions.

These algorithms can generate sample data matrices of any size from a given covariance structure. Assuming multivariate normality, the independent mean vectors can be added to these data for complete moment structure representation. Although the RAM basis of this exposition is relatively new, the general simulation procedure has been used by others (e.g., Dickinson \& Wherry, 1973; Kaiser \& Dickman, 1962; Sands, 1973).

\section{On-Line Scenario}

One of the most fundamental features of the plasmode approach is its interactive relation between models and data. The most fundamental feature of RAM is its graphic representation. Add these two together and it is easy to see that the most advantageous package for this approach is in an interactive graphic environment.

A research laboratory classroom (LABCLAS) can be used in a single- and/or multiple-entry terminal design. One of the terminals may be a control station for supervisory monitoring. Some form of graphic input device (pen on pad or CRT) is introduced for model input. Many additional graphic features, such as path width reflecting coefficient size strength and dashes representing negative signs, may be introduced also (cf. Levin, 1976). A direct internal translation by RAM Equation 2 can now be completed by simple matrix manipulations. This can be done quickly on a variety of machine configurations due to the one-step simplicity of the matrix operation. A multivariable data generation routine then internally accepts the RAM $W$ and generates $G$ samples of size $\mathrm{N}_{\mathrm{g}}$ or their corresponding moment characteristics. Some applications may require knowledge of the model by only a subset of the research team, such as the monitor. In any case, multiple samples of data from multiple models are easily and rapidly entertained during a single class session.

This represents a rather simplistic proposed scenario.
As it turns out, the major implementation problems are not with the on-line interaction or with the graphics. The next section illustrates that the product of an interactive effort and the necessary computer hardware for fully graphic interaction, although not used here, are already at hand.

\section{HUMAN ABILITY SYSTEMS AS A SUBSTANTIVE EXAMPLE}

Horn and McArdle (1979) present perhaps the most extensive example of systems simulation of this type. Much of their effort was focused on a critical evaluation of RAM/LISREL as a data-analytic tool rather than as a simulation device. The majority of the mathematical statements of this section are globally paraphrased from their report to illustrate some ideas that are realistically possible at present.

The basis of this simulation was to explicate a theory of both static structure and dynamic process in adult development for one of the most widely used tests of human intelligence, the Wechsler Adult Intelligence Scale (WAIS; Wechsler, 1955). Although many theoretical orientations can be used, a model for the WAIS was derived from the developmental theory of fluid $(G f)$ and crystallized $(G c)$ intelligence (Cattell, 1971; Horn, 1978; Horn \& Cattell, 1967). Many of the mathematical ideas resemble those offered by Nesselroade (1977). These theories were combined to simulate manifest WAIS means, variances, and correlations for multiple-age and cohort groups.

\section{Measurement Structure}

A statement of the causal relations between five first-level $G f / G c$ latent variables and the 11 manifest WAIS variables is provided in Figure 5. The width of each path arrow indicates its relative size (see key to figure) in the measurement structure. For present purposes of simulation for the WAIS, only a subset of the possible attribute concepts of $G f / G c$ theory was used. These concepts, which are indicated as common factors in several studies of the structure in broad samples of ability-test performances (for overview, see Horn, 1978), may be briefly described as follows:

(1) Crystallized intelligence (Gc)-A broad collection of abilities representing the extent to which an individ. ual has appropriated cultural knowledge for his own use. (2) Fluid intelligence $(G f)-A$ broad collection of abilities that do not require prior knowledge acquisition. These are also considered to develop largely indepen. dently from the systematic influences that characterize acculturation. (3) Short-term acquisition and retrieval $(S A R)-$ A broad collection of the abilities of becoming aware, maintaining attention, recording information, and retaining it for short periods of time (roughly 3-30 sec). (4) Tertiary storage and retrieval (TSR)-Abilities of 

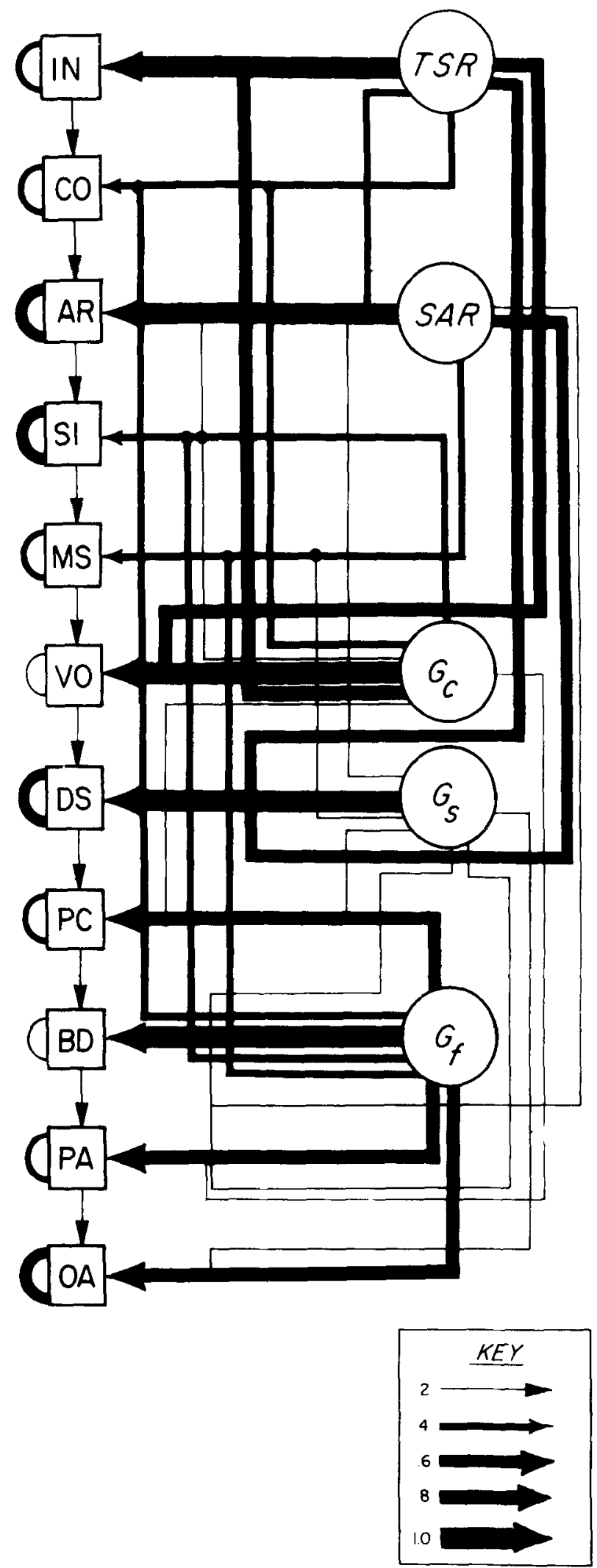

Figure 5. Measurement structure for the $G f / G c$ theory of the WAIS (from Horn \& McArdle, 1979). storing information in such a way that it is retained for long periods of time (minutes, months, years). (5) Broad speediness $(G s)$-Abilities of rapidly doing clerical and perceptual problems of a kind that all persons would solve, given enough time.

As can be seen in Figure 5, Gc is similar to the verbal scoring (sum of 1 to 6) and $G f$ is similar to the performance scoring (sum of 7 to 11) of the WAIS scales. But the differences are important. For instance, the tasks of memory span (MS) and arithmetic (AR; which is greatly dependent on memory) are not thought to be highly characteristic of $G c$. Additionally, $G f$ is thought to be implicated in the verbal test abilities of comprehension (CO), similarities (SI), and MS, as well as in the performance scales (excluding digit symbol; DS).

A variety of other relations were also postulated. The simulation of minor factors (Tucker, Koopman, \& Linn, 1969) was also used here for $S A R, T S R$, and $G s$. While these factors would be considered common in a broader battery, they serve here only to add increasing complexity to the manifest covariation seen in the WAIS. Another example is the traditional modeling of specific factors, reliably measured processes in each task that are not involved in any other tasks. The additional modeling of a Guttman (1954) simplex among the WAIS subtests (administered from top to bottom here) represents the hypothesis that systematic individual differences in learning, relaxing, becoming comfortable with the tests, and so on, operate to produce more or less enhancement in performance on Subtest $w_{i+j}$ as a consequence of prior experience with Subtest $w_{i}$. This first-order autoregressive effect is specific and small (.2), but it is definitely present.

One strong mathematical assumption of this overall theory is that the measurement model of Figure 5 is invariant for all groups of subjects at all times (cf. Meredith, 1964; Rozeboom, Note 2). The relations between factors at different times of measurement and their means and standard deviations can vary from age to age, cohort to cohort, or time to time, however.

\section{Higher Order Controls}

Figure 6 represents the notion that, while the attribute concepts of $G f / G c$ are causal variables relative to the effect subtest scores of the WAIS, they might be regarded as effects relative to causal factors at a higher level of abstraction. In particular, the model of Figure 6 helps to systematize parts of $G f / G c$ theory that stipulate that the ability organizations represented by $G f, G c, S A R, T S R$, and $G s$ are brought about by (1) neurological influences (NE), (2) acculturational learning influences $(A C)$, and (3) situational motivational influences $(S M)$.

Figure 6 illustrates how these higher order indirect influences can produce manifest relations. The $N E$ influence, for example, does not affect $G c$ directly. 


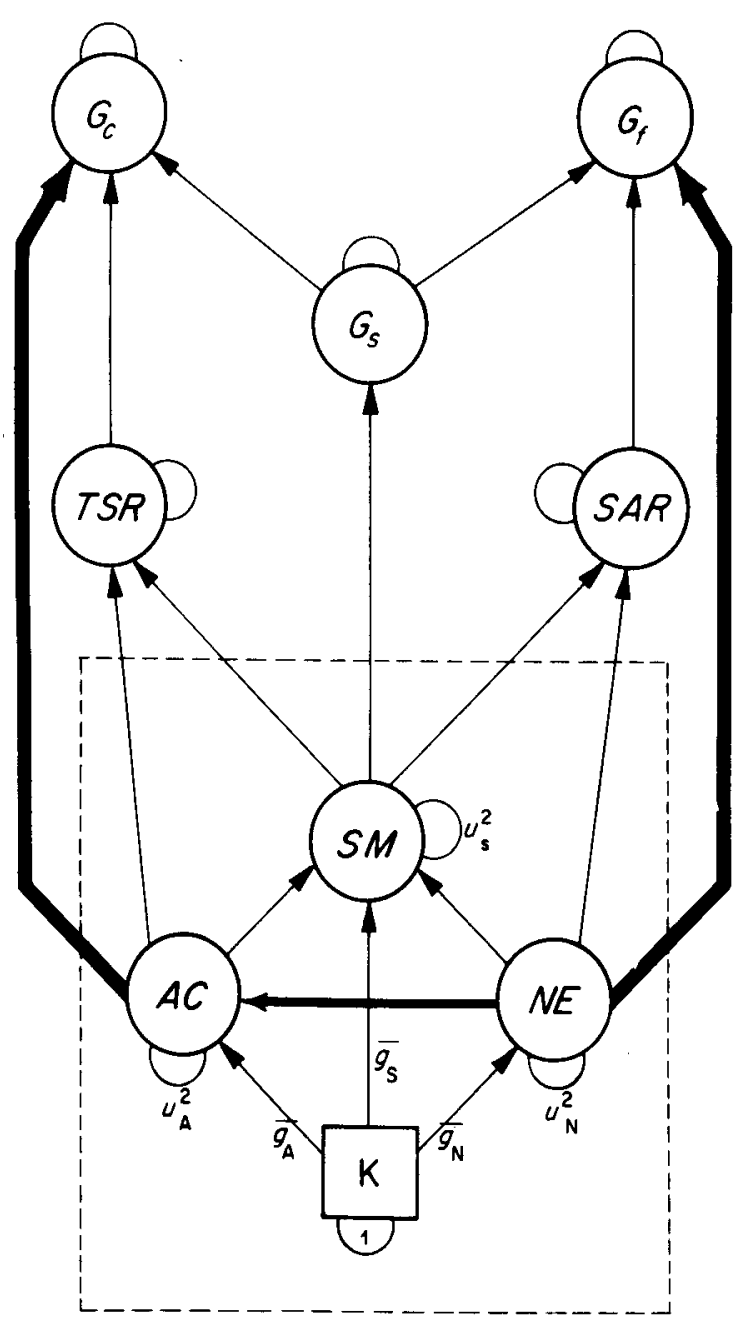

Figure 6. Higher order control of $G f / G c$ theory (controls inside dashed box) (from Horn \& McArdle, 1979).

It operates on $A C$ (at .4 ), which in turn has a major influence (.6) on $G c$. The path product of these two influences $(.4 \times .6=.24)$ gives a measure of the extent to which $G c$ is produced by $N E$ through the particular $N E-A C-G c$ pathway. $N E$ also affects $G c$ through several other pathways (e.g., $N E-S M-G s-G c$ ), so the overall effect can be considerable. Similarly, the $\bar{g}_{A}, \bar{g}_{S}$, and $\bar{g}_{\mathrm{N}}$ symbols represent the concept of specific means. That is, just as specific variance $\left(u_{\mathrm{A}}^{2}, u_{\mathrm{S}}^{2}\right.$, and $\left.u_{\mathrm{N}}^{2}\right)$ represents the idea that there can be reliable variation in $S M$ and $A C$ that is not accounted for by variation in $A C$ and $N E$, so the specific mean represents the idea that level on one variable is not accounted for by level on other variables.

The nomograph of Figure 6 represents a theory that fully accounts for the $G f, G c, G s, T S R$, and $S A R$ latent variables (and their interrelationships). Consequently, it also fully accounts for the phenotypic characteristics of the 11 WAIS subscore variables that are to be observed directly. The relationships depicted in the model of Figure 5, which was regarded as indicating latent vari- ables, are controlled according to theory by the six parameters of the model of Figure 6-namely, the specific variances and means of $N E, A C$, and $S M$. These high-level theoretical controlling mechanisms are isolated inside the dashed box of Figure 6.

\section{Dynamic Processes}

The basic influences represented by $N E, A C$, and $S M$ are considered to operate over time and circumstances (dynamically) to produce age, cohort, and time effects that would be manifested in $G f, G c, T S R, S A R$, and $G s$, and, subsequently, in the directly measured WAIS subscores. Such dynamic control at the high level is portrayed in graphic form in Figure 7. The size of the arrows from one circle to another represents the strength of a control or influence. $N E$ at age 30 years strongly (.8) controls $N E$ at age 40 years, but it only moderately (.4) influences $A C$ at age 30 years and even more weakly (.2) affects $S M$ at age 30 years. The single arrow from one WS (Wechsler scale variable) to another represents a model assumption that the specific component of a WAIS variable at one age controls in some small way (.2) the specific component of that same variable at the later age. For all of the variables, the influences are assumed to be the same over all of the 10-year aging intervals except that between 60 and 70 years of age. For this period, it is assumed that aging over a 5-year span is equivalent to the aging that occurs over a 10 -year span elsewhere (in the portion of adulthood considered in this study). The dynamic influences modeled for $60-70$ years are smaller (e.g., $N E_{60}-N E_{70}=.8 * .8=.64$ ) under an assumption that there are mediating variables at 65 years (represented as smaller circles).

Figure 8 extends the dynamics of Figure 7 by charting the mean changes for the basic controlling variables (of the theory) as these changes are dictated by the relationships shown in the previous features. Figure 8 indicates that in accounting for changes in the observed

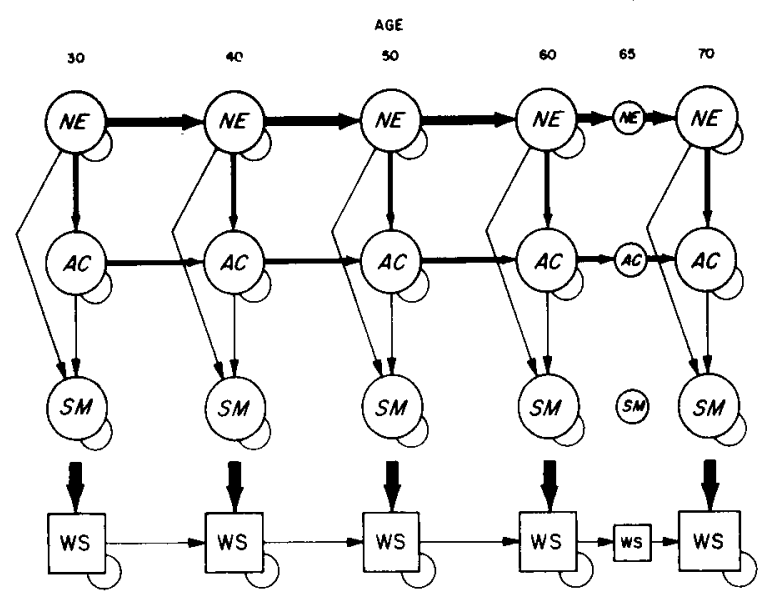

Figure 7. Dynamic process model for aging changes in the higher order control variables in $G f / G c$ theory (from Horn \& McArdle, 1979). 


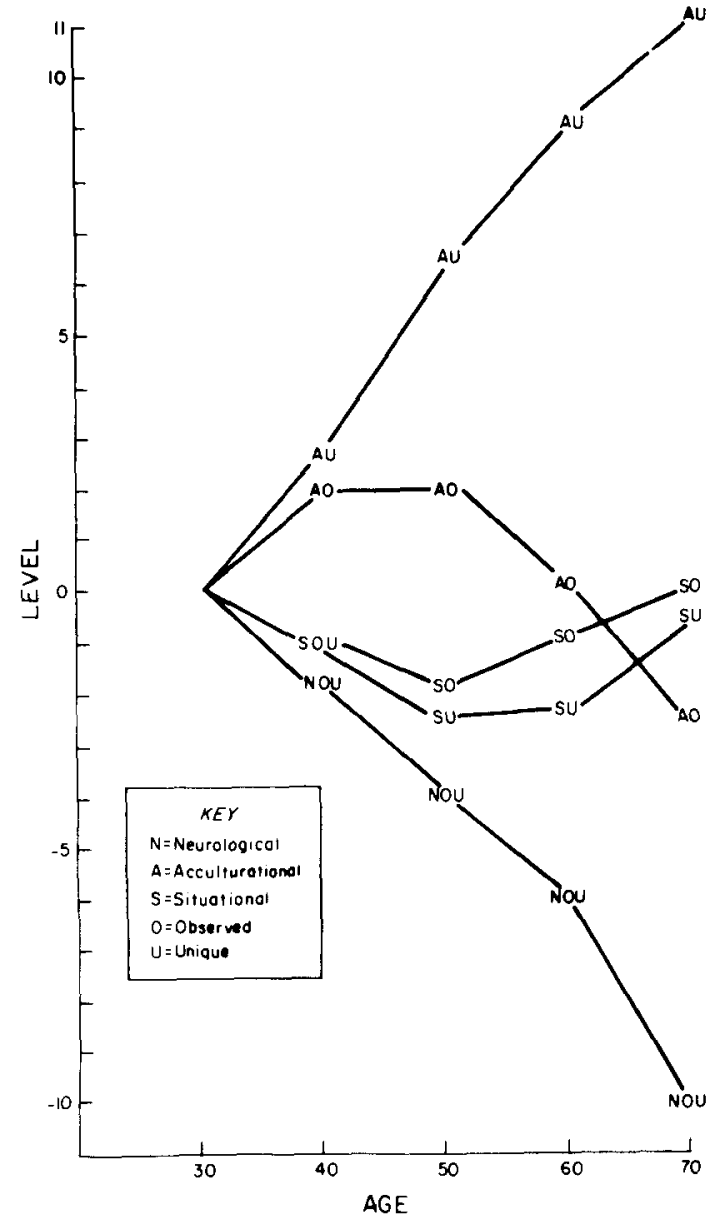

Figure 8. The dynamic mean process age change in the higher order control variables of $G f / G c$ theory (implied from Figure 8, Horn \& McArdle, 1979).

WAIS means, the specific $\overline{N E}, A C$, and $S M$, as well as causal influences expressed through other variables of the model, must be considered. Most important, the shift in the mean for $A C$ is affected by the causal influence of $N E$ (as depicted in Figures 6 and 7). Although the unique acculturation (AU) is modeled to continually increase (i.e., learning is continual), the causal effect of unique neurological (NU) tends to produce aging decline in observed acculturation ( $\mathrm{AO}$; i.e., continual learning is suppressed). In contrast, the model for $N E$ specifies that it is not affected by any other variable modeled in this network.

The principal purpose of these figures is to indicate that the mathematical model derived from $G f / G c$ theory, although considerably simplified relative to the model implied by more general statements of the theory (e.g., Horn, 1978), is quite a bit more complex than the verbal/performance model implied by the usual scoring of the WAIS. The inclusion of higher order controlling influences $N E, A C$, and $S M$ pushes $G f / G c$ theory up to a point at which the influences that yield the struc- ture and process outcomes of research are themselves modeled. The suggestion is that if $N E, A C$, and $S M$ were measured and their relationships were determined, this information could be used to reproduce results such as those that are modeled to depict the structure of the WAIS subscales or to describe the aging changes of $G f$ and $G c$.

\section{Data Generation}

This $G f / G c$ model, when specified in RAM terms, produces a distinct population-moment structure (means, variances, and correlations) for WAIS variables. The simulation performed generated a large data set based on additional considerations of the age, time, and cohort issues (cf. Horn \& Donaldson, 1977). Briefly, three cohorts were assumed to be born 10 years apart (in 1904, 1914, and 1924), so their underlying age model at a given time of testing utilized a different 3-decade sector of the general simulation model in Figures 7 and 8 . Other control parameters were also reset for each age and time. Data were then separately simulated for $\mathrm{N}=500$ for each of three cohorts measured at each of three times. Therefore, while the proposed moment data $W^{*}$ was of size $\mathrm{j}^{*}=34$ (11 WAIS at 3 times +1 constant), the $D^{*}$ and $U^{*}$ were of size $t^{*}=55(11+7$ at 3 times +1 constant $)$.

In order to add necessary realism, the resultant data were reset into appropriate boundaries (e.g., no scores < 0 ) and rescaled using the WAIS keys. Summary scores for full-scale IQ were calculated on the emerging profiles, and a selection mechanism was put into effect to simulate attrition. The lowest $20 \%$ of full-scale IQ scorers were eliminated for all ages, and the top $10 \%$ were eliminated from the early ages $(20,30$, and 40 years). In order to provide a realistic taste of this simulation, the resultant scale-scored data means are summarized $\left(\mathrm{N}_{\mathrm{g}}=160,128\right.$, and 80, respectively) in Figure 9. More details on these data and the follow-up LISREL-IV analyses are given by Horn and McArdle (1979).

The characteristic feature of this attempt at simulation is that a target data set is available: real WAIS means, variances, and intercorrelations for real subjects in this age range (Wechsler, 1955). The interactive creation of this data set was extensive but not formally monitored. This permitted the research team an opportunity to play with the valves over and over again until a fine-tuned, close-to-reality model was developed. The very essence of the task simultaneously required a broad perspective on substantive concepts and a healthy respect for the power of system controls. After numerous production runs under numerous control strategies, the substantively and mathematically appealing foundation presented here was obtained. While there still are problems in this "hydraulic system," directions for future improvement are now better understood. 


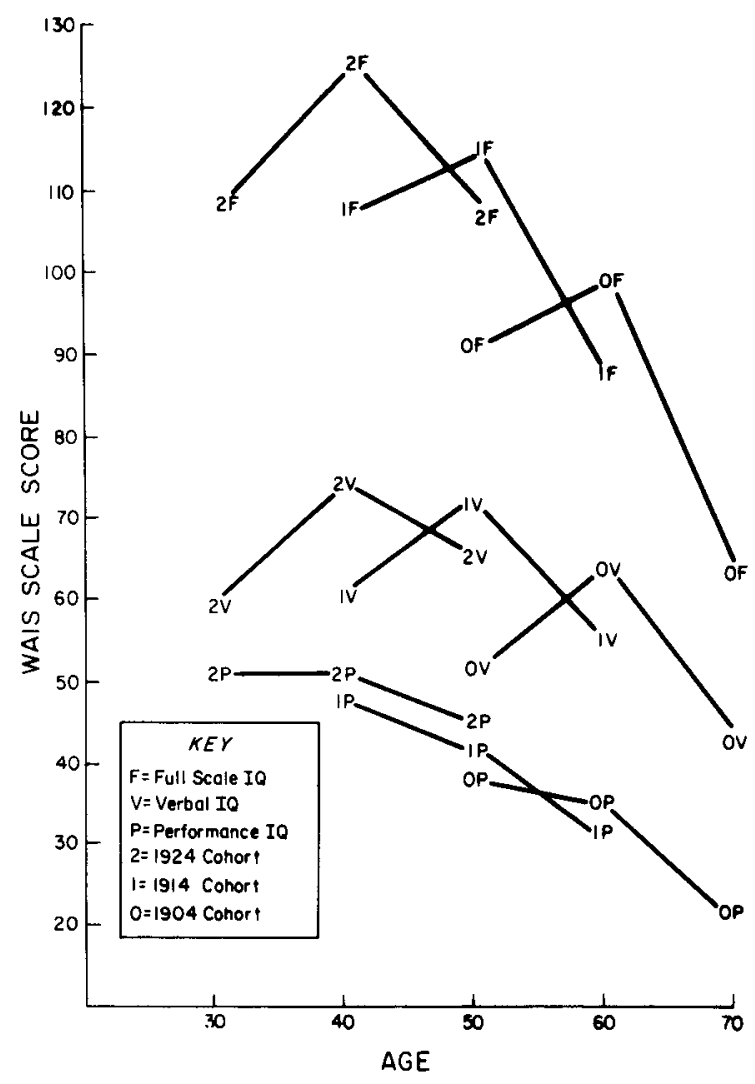

Figure 9. The observed WAIS means created by RAM simulation of $G f / G c$ theory for three cohort groups at three times of testing (from Horn \& McArdle, 1979).

\section{BROAD APPLICATIONS OF CAUSAL SIMULATION}

The causal systems modeling approach is not a new research tool. However, the ongoing mathematical developments in both causal analysis and multivariable simulation suggested here illustrate that its real power is dormant for a great many psychonomists. The intent of this final section is to briefly explore some possible applications of this approach, given its current status. The general guidelines provided by Cattell and Jaspers (1967, pp. 1-2) are still useful: "(a) to provide students with an exercise in mathematicostatistical analyses in which they can check their work at all stages against known values, (b) to evaluate the accuracy, fitness, biases, etc of various alternative procedures in mathematico-statistical analysis procedure, and (c) to stimulate intuitions about possible improvements in the mode itself in regard to its fitness to our scientific needs."

\section{Pedagogical Features}

One major virtue of this simulation enterprise is the broad training that it can provide. The exercise of designing a causal system for subsequent simulation requires an understanding of both multivariate theory and methods. As Lehman (1977, p. 11) suggests, "The simulation writer must make the function relating variables explicit, define initial values and parameters, and resolve contradictions, or the simulation cannot function properly." Of some importance here is the examination of alternative structural theories that produce exactly the same or similar observations. The possibility of teaching this in an interactive graphical fashion is clearly relevant. In this regard, RAM has proven useful in teaching structural analyses via linear structural relations (LISREL; Jöreskog \& Sörbom, 1978) methodology. For instance, notation used in the examples here provides a direct translation from the usual LISREL Greek and fully explicates many important LISREL concepts in nomographical terms (cf. McArdle, Note 1).

The classroom use of RAM simulation might typically begin by generating a single sample from a known causal structure. This can be done even for models that include complexities well beyond our current analytic capabilities (e.g., Horn \& McArdle, 1979). The teacher might serve as the monitor, with complete knowledge of the causal system at hand. Each student can be given a random sample from this known population. Then, the important exercise is to examine the problems involved in recovering the original system using alternative methods and models. This simulates the actual applied situation and closely follows the stimulating teaching conditions described and used by Lehman (1980). Good examples of this are also provided by Horn (1967), Sörbom (1975), Gustafsson and Lindstrom (Note 4), and Lohmöller (Note 5).

\section{Research Features}

Another very practical virtue of RAM simulation is its use as a permutation base for statistical tools of questionable validity. RAM can be used to create data from randomly designed or permuted alternative structures. Comparative study of these randomized baselines can prove useful to empirical evaluations. Good examples of this approach are provided by Heeler and Whipple (1976), Horn (1965), Horn and Knapp (1973), Nesselroade, Baltes, and Labouvie (1971), and Van Hemert (1976).

A more specific use of simulation is in the structural analysis of a given data set. In many cases, RAM/LISREL analysis (McArdle, Note 1) proves problematic. For instance, theoretical parameters may not all be identified, or a given problem may be too large (in terms of variables and/or parameters) for viable computer analysis. In these cases a Turing-type test between model and real data is perhaps still possible. This can be accomplished by using the population $W$ as "fixed" input starting values or with simulation $W$ vs. real data $W$ in a statistical equality test. Also, not all parameters need to be completely fixed. In the example provided here, the six theoretical controls could have been solved from given specific sets of available data. Information from the first derivatives might then show how the simulation 
should be altered (Saris, Pijper, \& Zewaart, 1978; Sorrbom, 1978). Although this requires extremely strong theory, and it has never been attempted in an actual example, it could prove to be a useful adjunct for analyses.

One important area of mathematical research is Monte Carlo simulation. Typically, this involves the very specific applications of thousands of simulation experiments (cf. McArdle, 1977). The use of this causal approach permits researchers to explicitly specify causal systems as the focus of an investigation. This permits meaningful comparisons of alternative models and methods and flexible design of the necessary population data. Examples of this approach include the studies of Mosbaek and Wold (1970), Tucker et al. (1969), Wackwitz and Horn (1971), and Boomsma (Note 6).

\section{Simulation Dialectics}

It should be obvious that this approach to simulation is not exactly equivalent to discrete-event simulations (cf. Lehman, 1977). These deterministic simulations begin with the description of a variety of steps or phases and the transitional probabilities associated with possible movement from one to the next. Bounded stochastic properties are introduced to create realism yet avoid microdescription of complete deterministic logic. Typically, the input is a set of initial conditions, and the output is a set of behaviors. The simulator can observe the individual and/or collective behavior of his "simula" at any phase and alter the model accordingly.

On the other hand, the concepts used in discreteevent simulation are closely analogous to the functional form advocated here. First, nomographs may easily be developed for a single subject measured many times (cf. Birkett \& Cattell, 1978). Alternatively, the functional process can be slowed down and examined only as subset joint-conditional probabilistic events. In this way, the value for a single outcome is based on the actual value of the input causes for that specific instance. In fact, the functional form of causal simulation can be considered a global outline for the long-term behavior of the discrete events. One immediate advantage for the functional approach is that it practically permits a very broad frame of reference. A functionally complete system may prove useful in the search for the more critical subsystems, which may then be further investigated by some more deterministic avenue.

The major conclusion, then, is that these simulation approaches walk hand in hand, each with its own special problems and virtues. The main differences lie in the level of observational reduction desired. While many of the simulations on intellectual development have been of a deterministic nature (e.g., Klahr, 1976), the example provided here (Horn \& McArdle, 1979) hopefully illustrates that the broad systems view has a useful place also.

It is important to put any form of simulation in proper perspective. The broad quest of psychonomy is to answer this question: "What are the causal laws of mental activity?" No simulation approach pretends to answer this question. Alternatively, simulation is valuable in that it does not foster the belief that advanced forms of multivariable statistical analyses provide magic potion for the seeker of these truths. The necessary baggage of causal statements, as explicitly defined earlier, usually proves to be much too heavy for most available observational knowledge. The process of generating data from a specified causal system illustrates that the detective work involved in trying to reverse this process, to infer causality from observation, has severe analytic limitations.

The most salient feature of this RAM approach to simulation is its direct relationship with available dataanalytic methodology. The practical benefit is that this represents a way of thinking about the relationship between theory and data. As theories are examined in a broad systems fashion (e.g., Cattell, 1971; Royce \& Buss, 1976), both benefits and problems can be more clearly seen from a state-of-the-art perspective. Therefore, while causal simulation provides no explicit rules for what to do, or a complete understanding of all possibilities, at least this view permits a peek at what is possible and what it may mean. Simulating is stimulating and can open up the doors of imagination in a psychonomically viable way.

\section{REFERENCE NOTES}

1. McArdle, J. J. Reticular analysis modeling (RAM) theory: The simplicity and generality of structural equations. Manuscript submitted for publication, October 1979.

2. Rozeboom, W. W. General linear dynamic analysis (GLDA). Unpublished manuscript, 1978.

3. Reichardt, C. Personal communication, October 1979.

4. Gustaffson, J. E., \& Lindstrom, B. Analyzing ATI data by structural analysis of covariance matrices. Paper presented at the annual meeting of the American Educational Research Association, San Francisco, April 1979.

5. Lohmöller, J. B. How longitudinal factor stability, continuity, differentiation, and integration are portrayed into the core matrix of three-mode factor analysis. Paper presented at the meeting of the European Psychometrics Society, Uppsala, Sweden, June 1978.

6. Boomsma, A. The robustness of a general method for the analysis of covariance structures against small sample sizes and departures from multivariate normality. Paper presented at the meeting of the European Psychometrics Society, Uppsala, Sweden, June 1978

\section{REFERENCES}

Ahrens, J. H., \& Dieter, U. Computer methods for sampling from the exponential and normal distributions. Communications of the $A C M, 1975,15,873-882$.

Baltes, P. R., Nesselroade, J. R., \& Cornelius, S. W. Multivariate antecedents of structural change in development: A simulation of cumulative environmental patterns. Multivariate Behavioral Research, 1978, 13, 127-152.

BentLer, P. M. The interdependence of theory methodology and empirical data: Causal modeling as an approach to construct validation. In D. B. Kandel (Ed.), Longitudinal research on drug abuse: Empirical findings and methodological issue. 
New York: Wiley, 1978.

Birkett, H., \& Cattell, R. B. Diagnosis of the dynamic roots of a clinical symptom by P-technique: A case of episodic alcoholism. Multivariate Experimental Clinical Research, 1978, 3, 173-194.

Bock, R. D. Multivariate statistical methods in behavioral research. New York: McGraw-Hill, 1975.

Bock, R. D., \& Bargmann, R. E. Analysis of covariance structures. Psychometrika, 1966, 31, 507-533.

CAtTEl, R. B. Handbook of multivariate experimental psychology. Chicago: Rand McNally, 1966.

CATtELl, R. B. Abilities: Their structure, growth and action. Boston: Houghton-Mifflin, 1971.

CATtELl, R. B. The scientific use of factor analysis in behavioral and life sciences. New York: Plenum Press, 1978.

Cattell, R. B., \& Jaspers, J. A general plasmode (No. 30-10-5-2) for factor analytic exercises and research. Multivariate Behavioral Research Monograph, No. 67-3, 1967.

Chambers, J. M. Computers in statistical research: Simulation and computer-aided mathematics. Technometrics, 1970, 12, $1-15$.

CoHen, J., \& Cohen, P. Applied multiple regression/correlation analysis for the behavioral sciences. New York: Wiley, 1975.

Dickinson, T. L., \& WherRY, R. J. A FORTRAN program for generating multiple samples of multivariate data with arbitrary population parameters. Educational and Psychological Measurement, 1973, 33, 715-718.

GutTman, L. The radex: A new approach to factor analysis. In P. Lazersfeld (Ed.), Mathematical thinking in the social sciences. New York: Columbia University Press, 1954.

Heeler, R. M., \& Whipple, T. W. A Monte Carlo aid to the evaluation of maximum likelihood factor analysis solutions. British Journal of Mathematical and Statistical Psychology, 1976, 29, 253-256.

HoRn, J. L. A rationale and test for the number of factors in factor analysis. Psychometrika, 1965, 30, 179-185.

Horn, J. L. On subjectivity in factor analysis. Educational and Psychological Measurement, 1967, 27, 811-820.

Horn, J. L. Human ability systems. In P. B. Baltes (Ed.), Life-span development and behavior. New York: Academic Press, 1978.

Horn, J. L., \& Cattell, R. B. Age differences in fluid and crystallized intelligence. Acta Psychologica, 1967, 26, 107-129.

Horn, J. L., \& Donaldoson, G. Faith is not enough: A response to the Baltes-Schaie claim that intelligence does not wane. American Psychologist, 1977, 32, 369-373.

HoRn, J. L., \& KNAPP, J. R. On the subjective character of the empirical base of Guilford's structure-of-intellect model. Psychological Bulletin, 1973, 80, 33-43.

Horn, J. L., \& McArdee, J. J. Perspectives on mathematical/ statistical model building (MASMOB) in research on aging. In L. W. Poon (Ed.), Aging in the 1980's: Selected contemporary issues in the psychology of aging. Washington, D.C: American Psychological Association, October 1979.

IMSL. International mathematical and statistical library (Vol. 7). Houston, Tex: 1979.

Jöreskog, K. G., \& Goldberger, A. S. Estimation of a model with multiple indicators and multiple causes of a single latent variable. Journal of the American Statistical Association, 1975, 70, 631-639.

Jöreskog, K. G., \& Sorbom, D. LISREL-IV: Analysis of linear structural relationships by the method of maximum likelihood. Chicago: National Educational Resources, 1978.

KaISER, H. F., \& Dickman, K. Sample and population score matrices and sample correlation matrices from an arbitrary population correlation matrix. Psychometrika, 1962, 27, 179-182.

KELLEY, H. H. The processes of causal attribution. American Psychologist, 1973, 28, 107-128.

KLAHR, D. Steps toward the simulation of intellectual development. In L. B. Resnick (Ed.), The nature of intelligence. New York: Wiley, 1976
LE HMAN, R. S. Computer simulation and modeling: An introduction. Hillsdale, N.J: Erlbaum, 1977.

LEHMAN, R. S. What simulation can do to the statistics and design course. Behavior Research Methods \& Instrumentation, $1980,12,157-159$.

LEViN, M. L. Displaying sociometric structures: An application of interactive computer graphics for instruction and analysis. Simulation and Games, 1976, 7, 295-310.

Lı, C. C. Path analysis. Pacific Grove, Calif: Boxwood Press, 1975.

MacWhinney, B. Limitations on the use of rules in describing behavior. In J. M. Scandura \& C. J. Brainerd (Eds.), Structural/ process models of complex human behavior. Amsterdam: Sijthoff \& Noordhoff, 1978.

Marsaglia, G. Random number generation. In A. Ralston \& C. L. Meek (Ed.), Encyclopedia of computer science. New York: Petrocelli/Charter, 1976.

MCARDLE, J. J. Empirical tests of multivariate generators. In D. C. Hoaglin \& R. E. Welsch (Eds.(, Proceedings of the Ninth Annual Symposium on the Interface between Computer Science and Statistics. Boston: Prindle, Weber, \& Schmidt, 1976.

McArdeE, J. J. The statistical analyses of Monte Carlo simulation data using the techniques of discrete multivariate analysis. In D. Hogben \& D. W. Fife (Eds.), Proceedings of the Tenth Annual Symposium on the Interface between Computer Science and Statistics. Washington, D.C: National Bureau of Standards, 1977.

McArdLe, J. J. A structural view of longitudinal repeated measures. In F. C. Leone (Ed.), Proceedings of the social sciences section of the American Statistical Association annual meeting. Washington, D.C: 1978.

McArdle, J. J. The development of general multivariate software. In J. J. Hirschbuhl (Ed.), Proceedings of the association for the development of computer-based instructional systems. Akron, Ohio: University of Akron, 1979.

McDonald, $\overline{\mathrm{R}}$. P. The structural analysis of multivariate data: A sketch of a general theory. Multivariate Behavioral Research, 1979, 14, 21-28.

Meredith, W. Notes on factorial invariance. Psychometrika, 1964, 29, 177-185.

Montanell, R. G. A computer program to generate sample correlations and covariance matrices. Educational and Psychological Measurement, 1975, 35, 195-197.

Mosваек, E. J., \& WolD, H. O. Interdependent systems. Structure and estimation. Amsterdam: North-Holland, 1970.

Nesselroade, J. R. Issues in studying developmental change in adults from a multivariate perspective. In J. E. Birren \& K. W. Schaie (Eds.), Handbook of the psychology of aging. New York: Van Nostrand Reinhold, 1977.

Nesselroade, J. R., Baltes, P. B., \& Labouvie, E. W. Evaluating factor invariance in oblique space: Baseline data generated from random numbers. Multivariate Behavioral Research, 1971, 1, 233-241.

Odell, P. L., \& Feiveson, A. H. A numerical procedure to generate a sample covariance matrix. Journal of the American Statistical Association, 1966, 61, 199-203.

RAPOPORT, A. The uses of mathematical isomorphism in general systems theory. In G. J. Klir (Ed.), Trends in general systems theory. New York: Wiley, 1972.

Royce, J. R., \& Buss, A. R. The role of general systems and information theory in multi-factor individuality theory. Canadian Psychological Review, 1976, 17, 1-21.

SANDS, W. A. A computer program for the generation of score vectors from a multivariate normal distribution with a specified mean vector and variance covariance matrix. Educational and Psychological Measurement, 1973, 33, 719-722.

Saris, W. E., Pijper, M., \& Zewaart, P. Detection of specification errors in linear structural equation models. In $\mathrm{K}$. F. Schuessler (Ed.), Sociological methodology, 1979. San Francisco: San Francisco: Jossey Bass, 1978.

Sörвom, D. A general method for studying differences in factor 
means and factor structure between groups. British Journal of Mathematical Statistical Psychology, 1974, 27, 229-239.

Sorвom, D. Detection of correlated errors in logitudinal data. British Journal of Mathematical and Statistical Psychology, 1975, 28, 138-151.

Sorkom, D. An alternative to the methodology for analysis of covariance. Psychometrika, 1978, 43, 381-396.

Suppes, P. A probabilistic theory of causalitv. Amsterdam: NorthHolland, 1970.

Thurstone, L. L. Multiple factor analysis. Chicago: University of Chicago Press, 1947.

Tucker, L. R., Koopman, R. F., \& LinN, R. L. Evaluation of factor analytic research procedures by means of simulated correlation matrices. Psychometrika, 1969, 34, 421-459.

van DEN WollenberG, A. L. Redundancy analysis. An alternative for canonical correlation analysis. Psychometrika, 1977,
42, 207-219.

Van Hemert, N. A. SIMRANTAR. A CDC-ALGOL60 simulation program to evaluate the goodness of fit in targeted rotation of factors. Tijdschrift woor Onderwijsresearch, 1976, 1(4), 172-184.

W Ackwitz, J. H., \& Horn, J. L. On obtaining the best estimates of factor scores. Multivariate Behavioral Research, 1971, 6, $389-408$

WeChSLer, D. Manual for the Wechsler Adult Intelligence Scale. New York The Psychological Corporation, 1955.

Wold, H. O. A. Causality and econometrics. Econometrika, 1954, 22, 162-177.

Wright, S. Correlation and causation. Journal of Agricultural Research, 1921, 20, 557-585.

Wright, S. The method of path coefficients. Annals of Mathematical Statistics, 1934, 5, 161-215. 